\section{Renal Impairment in Axial Spondyloarthritis: A Prevalent Condition to Consider}

\section{To the Editor:}

We read with interest the paper by Couderc, et al ${ }^{1}$ on the prevalence of renal impairment in patients with spondyloarthritis (SpA) from the ASASCOMOSPA study (Assessment of Spondyloarthritis international SocietyCOMOrbidities in SPondyloArthritis). Their results encouraged us to investigate renal impairment prevalence in our cohort. Renal impairment [estimated glomerular filtration rate (eGFR) $<60 \mathrm{ml} / \mathrm{min} / 1.73 \mathrm{~m}^{2}$ ] prevalence in the ASAS-COMOSPA cohort was $5.2 \%$ and was associated with age, HLA-B27 positivity, and inflammation in the multivariate analysis. Data are scarce on the prevalence of renal involvement in SpA with evident heterogeneity, ranging from $0.1 \%$ to $11.3 \%$ of the cases s $^{2,3,4,5,6,7,8}$.

Our cohort comprises 360 patients with axial SpA with data collected prospectively since their enrollment in an observational study at a tertiary rheumatology center. We performed a cross-sectional evaluation of data relating to renal function tests, inflammatory markers, demographics, disease activity measures, and concomitant cardiovascular disease from the last followup visit. Continuous data were compared regarding the presence of renal impairment using the Student $t$ test if the variables presented normal distribution (previous Shapiro-Wilk test), or Mann-Whitney U test where appropriate. Chi-square or Fisher's test was performed if the variable was categorical. Multivariate analyses were performed with logistic regression models including variables considered significant in the univariate analysis and clinically significant variables.

Data available from 339 patients were included, and showed a $12.1 \%$ (41 patients) prevalence of renal impairment (eGFR $<60 \mathrm{ml} / \mathrm{min} / 1.73 \mathrm{~m}^{2}$; Table 1). Age and male sex were significantly associated with renal impairment. The disease duration was longer in patients with renal impairment regardless of the age at diagnosis. Cardiovascular comorbidity, hypertension, and dyslipidemia were more frequently reported in these patients. Higher Ankylosing Spondylitis Disease Activity Score (ASDAS), Bath Ankylosing Spondylitis Functional Index (BASFI) score, and erythrocyte sedimentation rate were significantly associated with renal impairment, and although there was a similar tendency in the Bath Ankylosing Spondylitis Disease Activity Index, this did not reach statistical significance.

Regarding treatment, patients with renal impairment were reportedly taking nonsteroidal antiinflammatory drugs (NSAID) less often. In the multivariate model, age (OR 1.121, 95\% CI 1.054-1.191) and the presence of dyslipidemia (OR 6.241, 95\% CI 1.156-33.692) remained significantly associated to eGFR $<60 \mathrm{ml} / \mathrm{min} / 1.73 \mathrm{~m}^{2}$.

These results show a higher prevalence of renal impairment in our cohort when compared to the work of Couderc, et $a l^{1}$. This might be explained by a difference in disease duration between the cohorts, which was much longer in ours $(24.29 \pm 15.65 \mathrm{yrs})$ than in the ASAS-COMOSPA study (mean 8.6 $\pm 9 \mathrm{yrs}$ ), as well as our patients being older. Taking into account that disease duration and age are associated with renal impairment in our cohort, this may explain the disparity in prevalence.

Cardiovascular comorbidities were more frequently reported in the renal impairment group in both cohorts, although they did not remain statistically associated in the multivariate models. ASDAS and BASFI scores were higher in both cohorts in the eGFR $<60 \mathrm{ml} / \mathrm{min} / 1.73 \mathrm{~m}^{2}$ group, but were not associated in the multivariate analysis, highlighting that there is probably an influence from age and other cofounders. Other significant differences were the negative association of HLA-B27 positivity with renal impairment in the ASAS-COMOSPA cohort that we did not confirm in our cohort. This has only been reported in this paper and further confirmation is needed in other cohorts. We did also report lesser use of NSAID in the renal impairment group, which is more the consequence than the cause, because patients had their treatment adjusted as an outcome of the renal disease diagnosis. Unfortunately, data on the potential effect of the cumulative use of NSAID on renal impairment are not available in our cohort.

It is important to highlight that the prevalence of renal impairment can be significantly high in cohorts with longer SpA disease duration, and this needs to be taken into consideration when managing these patients.

Table 1. Comparative analysis of patients with renal impairment (RI) and recorded variables.

\begin{tabular}{lccc}
\hline Variables & RI, $\mathrm{n}=41$ & No RI, $\mathrm{n}=298$ & $\mathrm{p}$ \\
\hline Age, yrs, mean \pm SD & $71.12 \pm 7.8$ & $54.64 \pm 14.52$ & $<0.0001^{*}$ \\
Male, $\%$ & 87.8 & 71.1 & $0.024^{*}$ \\
Arterial hypertension, $\%$ & 82.9 & 26.5 & $<0.0001^{*}$ \\
Dyslipidemia, \% & 46.3 & 29.2 & $0.026^{*}$ \\
Diabetes mellitus, $\%$ & 19.5 & 9.7 & 0.103 \\
BMI, mean \pm SD & $28.45 \pm 5.72$ & $26.36 \pm 4.39$ & 0.065 \\
Smokers, $\%$ & 69.7 & 56.9 & 0.159 \\
HLA-B27+, $\%$ & 80 & 84.2 & 0.5 \\
axSpA phenotype: AS-nr-axSpA, \% & $100-0$ & $91.6-8.4$ & 0.056 \\
Disease duration, yrs, mean \pm SD & $34.83 \pm 13.71$ & $22.84 \pm 15.35$ & $<0.0001^{*}$ \\
Age at diagnosis, yrs, median (IQR) & $33(22)$ & $30 \pm 16$ & 0.138 \\
NSAID $>$ 25\%, $\%$ & 22 & 36.4 & $0.003^{*}$ \\
Biologic use, $\%$ & 24.4 & 27.9 & 0.641 \\
BASDAI, mean \pm SD & $4.005 \pm 2.1$ & $3.5 \pm 2.13$ & 0.156 \\
BASFI, mean \pm SD & $5.2 \pm 2.56$ & $3.5 \pm 2.61$ & $<0.0001^{*}$ \\
ASDAS-CRP, mean \pm SD & $2.57 \pm 0.87$ & $2.22 \pm 0.92$ & $0.04^{*}$ \\
ASDAS-ESR, mean \pm SD & $2.66 \pm 0.9$ & $2.23 \pm 0.87$ & $0.01^{*}$ \\
CRP, median (IQR) & $3.8(6.65)$ & $3.0 \pm 5.65$ & 0.052 \\
ESR, median (IQR) & $15(23.3)$ & $8 \pm 13$ & $0.008^{*}$ \\
\hline
\end{tabular}

* p < 0.05. BMI: body mass index; axSpA: axial spondyloarthritis; nr-axSpA: nonradiographic axSpA; AS: ankylosing spondylitis; IQR: interquartile range; NSAID: nonsteroidal antiinflammatory drugs; BASDAI: Bath Ankylosing Spondylitis Disease Activity Index; BASFI: Bath Ankylosing Spondylitis Functional Index; ASDAS-CRP: Ankylosing Spondylitis Disease Activity Score using C-reactive protein; ASDAS-ESR: ASDAS using erythrocyte sedimentation rate. 


\section{ACKNOWLEDGMENT}

We thank Dr. Helena Marzo-Ortega for critical analysis of the final manuscript.

XABIER MICHELENA @ , MD, Hospital Universitari de Bellvitge-IDIBELL, Hospitalet de Llobregat, and Leeds Institute of Rheumatic and Musculoskeletal Medicine, University of Leeds, and UK National Institute for Health Research Leeds Biomedical Research Centre, Leeds Teaching Hospitals Trust, Leeds, UK; CARLA MARCO- PASCUAL (1), MD, Hospital Universitari de Bellvitge-IDIBELL, Hospitalet de Llobregat; XAVIER GONZÁLEZ-GIMÉNEZ [1, MD, Hospital Universitari de Bellvitge-IDIBELL, Hospitalet de Llobregat; XAVIER JUANOLA ${ }^{\circledR}$, MD, $\mathrm{PhD}$, Hospital Universitari de Bellvitge-IDIBELL, Hospitalet de Llobregat, Barcelona, Spain. Address correspondence to Dr. X. Juanola,

Rheumatology Department, Hospital Universitari de Bellvitge,

C/ Feixa Llarga, s/n. 08907, L'Hospitalet de Llobregat, Barcelona, Spain.

E-mail: xjuanola@bellvitgehospital.cat

\section{REFERENCES}

1. Couderc M, Pereira B, Molto A, Tiple A, Soubrier M, Dougados M. The prevalence of renal impairment in patients with spondyloarthritis: results from the international ASAS-COMOSPA study. J Rheumatol 2018;45:795-801.

2. Wu Y, Zhang G, Wang N, Xue Q. Risk factors of renal involvement based on different manifestations in patients with ankylosing spondylitis. Kidney Blood Press Res 2018;43:367-77.
3. Rodrigues CE, Vieira WP, Bortoluzzo AB, Goncalves CR, da Silva JA, Ximenes AC, et al. Low prevalence of renal, cardiac, pulmonary, and neurological extra-articular clinical manifestations in spondyloarthritis: analysis of the Brazilian Registry of Spondyloarthritis. Rev Bras Reumatol Engl Ed 2012;52:375-83.

4. Kristensen LE, Jakobsen AK, Askling J, Nilsson F, Jacobsson LT. Safety of etoricoxib, celecoxib, and nonselective nonsteroidal antiinflammatory drugs in ankylosing spondylitis and other spondyloarthritis patients: a Swedish national population-based cohort study. Arthritis Care Res 2015;67:1137-49.

5. Ben Taarit C, Ajlani H, Ben Moussa F, Ben Abdallah T, Ben Maiz $\mathrm{H}$, Khedher A. Renal involvement in ankylosing spondylitis: concerning 210 cases. Rev Med Interne 2005;26:966-9.

6. Samia B, Hazgui F, Abdelghani KB, Hamida FB, Goucha R, Hedri $\mathrm{H}$, et al. Renal abnormalities in ankylosing spondylitis. Nephrol Ther 2012;8:220-5.

7. Lee SH, Lee EJ, Chung SW, Song R, Moon JY, Lee SH, et al. Renal involvement in ankylosing spondylitis: prevalence, pathology, response to TNF-a blocker. Rheumatol Int 2013;33:1689-92 .

8. Levy AR, Szabo SM, Rao SR, Cifaldi M, Maksymowych WP. Estimating the occurrence of renal complications among persons with ankylosing spondylitis. Arthritis Care Res 2014;66:440-5

First Release November 1 2019; J Rheumatol 2020;47:2; doi:10.3899/jrheum.191016 